CLINICAL STUDY

\title{
Smoking as a risk factor for thyroid volume progression and incident goiter in a region with improved iodine supply
}

\author{
Till Ittermann ${ }^{1,4}$, Carsten Oliver Schmidt ${ }^{1}$, Axel Kramer ${ }^{2}$, Harald Below ${ }^{2}$, Ulrich John ${ }^{3}$, Michael Thamm ${ }^{6}$, \\ Henri Wallaschofski ${ }^{5}$ and Henry Völzke ${ }^{1}$ \\ ${ }^{1}$ Institute for Community Medicine, Ernst Moritz Arndt University, Walther-Rathenau-Straße 48, D-17487 Greifswald, Germany, ${ }^{2}$ Institute of Hygiene \\ and Environmental Medicine, ${ }^{3}$ Institute of Epidemiology and Social Medicine, ${ }^{4}$ Institute of Clinical Chemistry and Laboratory Medicine and ${ }^{5}$ Department of \\ Gastroenterology, Endocrinology and Nutrition, University of Greifswald, Greifswald, Germany and ${ }^{6}$ Robert-Koch-Institute, Berlin, Germany \\ (Correspondence should be addressed to T Ittermann; Email: till.ittermann@uni-greifswald.de)
}

\begin{abstract}
Objective: The role of smoking in the pathogenesis of thyroid enlargement is currently under debate. It has been hypothesized that the effect of smoking on increased thyroid volume is larger in regions with than in regions without iodine deficiency. The aim of this paper was to investigate the association of smoking with thyroid volume progression and incident goiter for different age-strata in a region with improved iodine supply.

Design and methods: The population-based Study of Health in Pomerania compromised 3300 subjects with complete 5-year examination follow-up. Data from 2484 participants without known history of thyroid disorder or thyroid medication were analyzed. Thyroid size was evaluated by ultrasound. Determinants of thyroid volume progression and incident goiter, i.e., newly occurred goiter between baseline and follow-up, were analyzed by linear and logistic regression respectively.

Results: Participants aged 20-39 years who were current smokers at baseline and at follow-up had a lower risk of incident goiter (odds ratio: 0.33; 95\% confidence interval (CI): $0.15 ; 0.71 ; P=0.005$ ). In this subpopulation, age was inversely related to thyroid volume progression. In subjects aged 60-79 years, smoking at baseline and follow-up was a risk factor for thyroid volume progression $(\beta$ : 3.37; 95\% CI: 0.84; 5.89; $P=0.009)$. After exclusion of individuals who had actual goiter in ultrasound at baseline, this association disappeared.

Conclusion: We conclude that the inverse association between smoking and goiter in young adults and the lacking association of smoking with goiter and thyroid volume progression in adult non-goitrous subjects indicate that smoking has a declining impact on thyroid growth in the study region. Our findings mirror the improved iodine supply of Northeast Germany.
\end{abstract}

European Journal of Endocrinology 159 761-766

\section{Introduction}

The role of smoking in the pathogenesis of thyroid enlargement is currently under debate. It has been hypothesized that the effect of smoking on increased thyroid volume is larger in regions with than in regions without iodine deficiency (1-4). Previous studies $(5,6)$, which have been conducted in iodine-deficient areas, detected smoking as a risk factor for thyroid enlargement. Other studies (7), however, did not confirm this finding. Evidence from regions with sufficient iodine supply is likewise conflicting. Some studies $(8,9)$ detected an association between smoking and thyroid enlargement, while others $(10,11)$ did not. Additionally, a twin study that recruited participants from regions with and without sufficient iodine supply detected smoking as a risk factor for goiter (12). Most of the previous studies are limited by their crosssectional $(1,4-12)$ or retrospective design (13).
Methodical constraints, such as selection or recall bias, might have additionally impaired results. Only one study (3) used a prospective cohort design, but was conducted in a selected population of parous women. Therefore, we sought to investigate the association between smoking and thyroid growth in a populationbased prospective cohort study.

The improved supply of iodine salt into food productions and individual salt consumption during the 1990s in the study region of Northeast Germany led us to the paradoxical situation of high goiter prevalence in a region of improved iodine supply (14). Previous studies (15) investigated the difference in thyroid growth between regions of mild and moderate iodine deficiency in different age strata. Especially, younger subjects should show more benefit from iodine improvement than elder subjects. Therefore, we investigated the association of smoking with thyroid volume progression and incident goiter in different age strata. 


\section{Materials and methods}

\section{Study subjects}

The Study of Health in Pomerania (SHIP) is a populationbased study in West Pomerania, a region in the north-east of Germany including the three cities Greifswald, Stralsund, Anklam, and 29 surrounding communities (16). The total population comprised 212157 inhabitants. As in most parts of Germany, West Pomerania is a region of former iodine deficiency (14), which resulted in a high prevalence of goiter (6). During the 1990s, improved iodine supplementation has normalized this deficiency level resulting in a median iodine excretion value of $124 \mu \mathrm{g} / \mathrm{l}(14)$. Currently, in the KiGGS study, a German survey in children and adolescents, a median iodine excretion of $117 \mu \mathrm{g} / \mathrm{l}$ was detected (17). SHIP and KiGGS argue for an improvement of iodine excretion levels in Germany on a lower recommended level.

For the SHIP baseline study, a sample from the population aged 20 to 79 years was drawn. Selection of the sample was done using population registries and performed in two steps. In Germany, all residents have to be registered. First, the three cities of the region (with 17 076-65977 inhabitants) and 12 towns (with 1516-3044 inhabitants) were selected, and then 17 out of 97 smaller towns (with less than 1500 inhabitants) were drawn at random. Secondly, from each of the selected communities, subjects were drawn at random, proportional to the population size of each community, and stratified by age and gender. Only individuals with German citizenship and main residency in the study region were included. Finally, 7008 subjects were sampled, with 292 persons of each gender in each of the twelve 5-year age strata. The net sample (without migrated or deceased persons) comprised 6267 eligible subjects. Selected persons received a maximum of three written invitations. In case of nonresponse, letters were followed by a phone call or by home visits if contact by phone was not possible. The population of the baseline SHIP finally comprised 4310 participants (2117 men and 2193 women) corresponding to a final response of $68.8 \%$ (16). Baseline examinations were conducted between 1997 and 2001. Between 2002 and 2006, all participants were re-invited to take part in an examination follow-up, in which 3300 subjects took part (1589 men and 1711 women; $83.5 \%$ of all eligible subjects). The median follow-up time was 5.00 years (minimum, 4.42 years; maximum, 8.58 years; 17314.7 person years). All participants gave informed written consent. The study followed the recommendations of the Declaration of Helsinki and was approved by the Ethics Committee of the University of Greifswald.

Among the participants, 77 (33 men and 44 women) had missing data in one or more of the variables involved in the data analysis, 717 (218 men and 499 women) had self-reported known thyroid disorders at baseline or follow-up, and 58 (14 men and 44 women) received thyroid therapy at baseline or follow-up. We excluded these 852 individuals $(265$ men and 587 women) from further analysis, resulting in a study population of 2448 (1324 men and 1124 women) participants. When using incident goiter as the dependent variable, we excluded a further 801 subjects (468 men and 333 women) with goiter at baseline. This resulted in a population of 1647 participants (856 men and 791 women).

\section{Assessments}

Socio-demographic characteristics, smoking, and history of known thyroid diseases or use of antithyroid medication were assessed by computer-aided personal interviews. According to cigarette smoking status, participants were categorized into five groups (1, never smoker; 2 , smoker at both baseline and follow-up; 3, smoker at follow-up but not at baseline; 4, smoker at baseline but not at follow up; 5, nonsmoker at baseline and follow-up but former smoker). Former smokers were individuals who had smoked during their lifetime but not in the last 12 months prior to the time of the baseline examination. Pack years for current and former smokers were calculated by multiplying the duration of smoking in years with the amount of packs (20 cigarettes were defined as one pack) an individual smoked a day. Pack years were divided into three categories (1, 0 pack years; 2 , \# pack years < median pack years of former and current smokers; 3, \# pack years $>$ median pack years of former and current smokers). Height and weight were measured for the calculation of the body mass index: $\mathrm{BMI}=$ weight $(\mathrm{kg}) /$ height $^{2}\left(\mathrm{~m}^{2}\right)$.

Iodine concentrations were measured by urine iodine excretion. Spot urine samples of the participants were collected and analyzed for iodine and creatinine concentration by photometric procedure (Photometer ECOM 6122, Eppendorf, Hamburg, Germany) with Sandell and Kolthoff reaction (18). The iodine-creatinine ratio was calculated by dividing urinary iodine by urinary creatinine concentration.

Blood samples were analyzed in one central laboratory. Serum thyrotropin (TSH), free triiodothyronine $\left(\mathrm{FT}_{3}\right)$, and free thyroxine $\left(\mathrm{FT}_{4}\right)$ levels were determined by immunochemiluminescent procedures $\left(\mathrm{FT}_{3}\right.$, LUMItest, Brahms, Berlin, Germany; TSH and $\mathrm{FT}_{4}$, LIA-mat, Byk Sangtec Diagnostica GmbH, Frankfurt, Germany).

Thyroid ultrasonography was performed in both examinations with an Ultrasound VST-Gateway, with a $5 \mathrm{MHz}$ linear array transducer (Diasonics, Santa Clara, CA, USA). The normal thyroid echo pattern was classified as homogeneous. A homogeneous echo pattern with reduced echogenicity was defined as hypoechogenic. Thyroid volume was calculated as length $\times$ width $\times$ depth $\times 0.479(\mathrm{ml})$ for each lobe (19). The intra- and inter-observer reliabilities were assessed before the start of the study and afterwards semi-annually during the study. 
All measurements of the thyroid volume showed Spearman correlation coefficients of $>0.85$ and mean differences $( \pm 2$ S.D. $)$ of the mean bias of $<5 \%(<25 \%)$. Thyroid volume progression was defined as difference between thyroid size at follow-up and baseline. Goiter was defined as a thyroid volume $>18 \mathrm{ml}$ in women and $>25 \mathrm{ml}$ in men (20).

\section{Statistical analysis}

Data on quantitative characteristics are expressed as median and inter-quartile range. Data on qualitative characteristics are expressed as percent values or absolute numbers, as indicated. The study population was divided into three groups according to the presence or absence of goiter at baseline and follow-up (1, presence of goiter at baseline; 2, absence of goiter at baseline and follow-up; 3, absence of goiter at baseline but presence at follow-up). Comparisons between groups were made using $\chi^{2}$ test (qualitative data) or Wilcoxon test (quantitative data). Wilcoxon's signed rank test was used for paired data. Determinants of thyroid volume change and incident goiter were analyzed by linear and logistic regression respectively. All models were adjusted for age, gender, and body mass index. In the first step, both analyses were performed separately for three different age strata (20-39, 40-59, and 60-79 years). In the second step, analyses were performed for the whole population, and interactions between the smoking variables and age were tested. Interactions were kept in the models for $P$ values $<0.1$. For all interactions, the $\beta$ and its s.E.M. are outlined. From linear regression models, the $\beta$ and its 95\% confidence interval $(95 \% \mathrm{CI})$ and from logistic regression, odds ratio, and its $95 \% \mathrm{CI}$ are given. A value of $P<0.05$ was considered statistically significant. All statistical analyses were performed with SAS 9.1 (SAS Institute, Inc., Cary, NC, USA).

\section{Results}

Among the 1647 participants without goiter at baseline, 291 (151 men and 140 women) individuals developed goiter during follow-up (5-year incidence: 17.7\%). Individuals who developed goiter during followup had lower serum TSH levels, were elder, more often overweight, less often current smokers but more often former smokers, and reported more pack years than subjects who had no goiter at baseline and follow-up (Table 1). Compared with subjects without goiter at both baseline and follow-up, individuals with goiter at baseline were elder, more often smokers, less often females, had a higher BMI and lower levels of serum $\mathrm{TSH}$, but higher levels of $\mathrm{FT}_{3}$. Current or former smokers with goiter at baseline had more pack years than smokers with no goiter at baseline and follow-up. Ever smokers at baseline had an improved risk of goiter compared with never smokers at baseline (relative risk: 1.15 ; $95 \%$ CI: $1.08,1.21)$. In all three groups, iodinecreatinine ratio decreased during follow-up. In the whole selected population, the median iodine-creatinine ratio decreased from $133.9 \mu \mathrm{g} / \mathrm{g}(99.8 \mu \mathrm{g} / \mathrm{g} ; 179.8 \mu \mathrm{g} / \mathrm{g})$ at baseline to $128.1 \mu \mathrm{g} / \mathrm{g}(89.9 \mu \mathrm{g} / \mathrm{g} ; 178.3 \mu \mathrm{g} / \mathrm{g})$ at follow-up $(P<0.001)$.

Table 2 shows the results of the age-specific linear regression with thyroid volume progression as outcome. Subjects who were smokers at baseline and follow-up in

Table 1 Characteristics of the study population with and without development of goiter.

\begin{tabular}{|c|c|c|c|c|c|}
\hline & $\begin{array}{c}\text { No goiter at baseline } \\
\text { and follow-up } \\
(n=1356)\end{array}$ & $\begin{array}{l}\text { Goiter at baseline } \\
\qquad(n=801)\end{array}$ & $\begin{array}{l}\text { Goiter at follow-up } \\
\text { only }(n=291)\end{array}$ & $\boldsymbol{P}^{\mathrm{a}}$ & $P^{\mathrm{b}}$ \\
\hline Age (baseline) (years) & $45(32 ; 57)$ & $52(41 ; 64)$ & $53(40 ; 64)$ & $<0.001$ & $<0.001$ \\
\hline Thyroid volume (baseline) (ml) & $14.84(12.0 ; 18.0)$ & $27.40(22.7 ; 32.6)$ & $17.81(15.8 ; 22.4)$ & $<0.001$ & $<0.001$ \\
\hline Thyroid volume (follow-up) (ml) & $15.44(12.5 ; 18.6)$ & $26.65(21.4 ; 34.4)$ & $25.43(19.9 ; 27.8)$ & $<0.001$ & $<0.001$ \\
\hline $\begin{array}{l}\text { Urinary iodine creatinine-ratio (baseline) } \\
(\mu \mathrm{g} / \mathrm{g})\end{array}$ & $131.0(98.2 ; 176.5)$ & $138.2(101.4 ; 186.8)$ & $138.1(101.9 ; 178.5)$ & 0.216 & 0.106 \\
\hline$\Delta$ Urinary iodine-creatinine ratio $(\mu \mathrm{g} / \mathrm{g})$ & $-3.6(-58.8 ; 44.7)$ & $-3.9(-57.6 ; 38.9)$ & $-2.9(-57.8 ; 48.3)$ & 0.543 & 0.788 \\
\hline Body mass index (baseline) $\left(\mathrm{kg} / \mathrm{m}^{2}\right)$ & $25.71(22.9 ; 28.8)$ & $27.68(25.0 ; 30.7)$ & $27.87(24.7 ; 30.5)$ & $<0.001$ & $<0.001$ \\
\hline Gender (men) & $705(52.0 \%)$ & $468(58.43 \%)$ & $151(51.9 \%)$ & 0.004 & 0.975 \\
\hline \multicolumn{6}{|l|}{ Cigarette smoking } \\
\hline Never smoker & $555(40.9 \%)$ & $252(31.46 \%)$ & $120(41.2 \%)$ & \multirow[t]{5}{*}{$<0.001$} & \multirow[t]{5}{*}{$<0.001$} \\
\hline Smoker before baseline & $416(30.7 \%)$ & $284(35.46 \%)$ & $113(38.8 \%)$ & & \\
\hline Smoker at baseline only & $73(5.4 \%)$ & $52(6.49 \%)$ & $17(5.8 \%)$ & & \\
\hline Smoker at follow-up only & $63(4.7 \%)$ & $24(3.00 \%)$ & $9(3.1 \%)$ & & \\
\hline Smoker at baseline and follow-up & $249(18.4 \%)$ & $189(23.60 \%)$ & $32(11.0 \%)$ & & \\
\hline Pack years & $11.1(5.5 ; 23.8)$ & $17.3(9.4 ; 29.0)$ & $15.0(6.7 ; 28.1)$ & $<0.001$ & 0.019 \\
\hline Serum thyrotropin (baseline) (mU/l) & $0.79(0.57 ; 1.10)$ & $0.53(0.36 ; 0.76)$ & $0.61(0.42 ; 0.83)$ & $<0.001$ & $<0.001$ \\
\hline Free triiodothyronine (baseline) (pmol/l) & $5.26(4.72 ; 5.78)$ & $5.20(4.70 ; 5.80)$ & $5.24(4.80 ; 5.80)$ & 0.680 & 0.709 \\
\hline Free thyroxin (baseline) (pmol/l) & $12.40(10.80 ; 13.90)$ & $12.70(11.10 ; 14.36)$ & $12.32(10.58 ; 14.20)$ & 0.005 & 0.996 \\
\hline
\end{tabular}

Data are given as numbers (percentage) or median (25th and 75th percentile). $\Delta$ Change between baseline and follow-up.

${ }^{a} \chi^{2}$ test (qualitative data) and Wilcoxon test (quantitative data) for row one and two.

${ }^{b} \chi^{2}$ test (qualitative data) and Wilcoxon test (quantitative data) for row one and three. 
Table 2 Risk factors for thyroid volume progression.

\begin{tabular}{lcrr}
\hline & $\begin{array}{c}\mathbf{2 0 - 3 9} \text { years } \beta \\
(\mathbf{9 5 \%} \text { confidence interval) }\end{array}$ & $\begin{array}{c}\mathbf{4 0 - 5 9} \text { years } \beta \\
(95 \% \text { confidence interval) }\end{array}$ & $\begin{array}{c}\mathbf{6 0 - 7 9} \text { years } \beta \\
(\mathbf{9 5 \%} \text { confidence interval) }\end{array}$ \\
\hline Smoker at baseline and follow-up & $-0.54(-1.18 ; 0.08)$ & $-0.46(-1.42 ; 0.50)$ & $3.37(0.84 ; 5.89)^{*}$ \\
Smoker at baseline only & $0.12(-0.88 ; 1.12)$ & $0.48(-1.06 ; 2.03)$ & $-0.31(-3.10 ; 2.47)$ \\
Smoker at follow-up only & $-0.47(-1.55 ; 0.62)$ & $-0.75(-2.61 ; 1.10)$ & $-0.69(-5.22 ; 3.83)$ \\
Smoker only before baseline & $-0.25(-0.93 ; 0.44)$ & $0.62(-0.20 ; 1.45)$ & $0.93(-0.43 ; 2.29)$ \\
Age (years) & $-0.10(-0.15 ;-0.05)^{\star}$ & $0.01(-0.05 ; 0.07)$ & $0.03(-0.06 ; 0.12)$ \\
Gender (ref. female) & $0.34(-0.18 ; 0.86)$ & $-0.56(-1.26 ; 0.14)$ & $0.20(-1.13 ; 1.52)$ \\
Body mass index $\left(\mathrm{kg} / \mathrm{m}^{2}\right)$ & $0.06(-0.01 ; 0.12)$ & $0.05(-0.02 ; 0.12)$ & $0.12(-0.01 ; 0.26)$ \\
\hline
\end{tabular}

Linear regression for thyroid volume progression as outcome. Reference group for smokers: non-smokers. ${ }^{\star} P<0.05$.

the elder subgroup (60-79 years) had a higher risk of thyroid volume progression than nonsmokers. However, after exclusion of individuals with goiter at baseline, this association disappeared ( $\beta$ : 1.64; 95\% CI: - 1.20, 4.48; $P=0.256)$. An interaction between smoking and age $\geq 60$ years was detected after including two dichotomous age variables (I, $4-59$ vs $<40$ years; II, $\geq 60$ vs $<40$ years) and their interaction terms with smoking into the model $(\beta=3.50$, s.E.M. $=0.97, P=0.001)$.

Table 3 outlines the results of the logistic regression with incident goiter as outcome for the three age strata. In the population $<40$ years, smoking at baseline and follow-up was inversely associated with goiter. Considering one logistic model over the whole population revealed an interaction between age and smoking $(\beta=0.03$, S.E.M. $=0.02, P=0.055)$. For clarifying this interaction, a logistic model with two dichotomous age variables (I, $40-59$ vs $<40$ years; II, $\geq 60$ vs $<40$ years) was performed. This calculation revealed significant interactions of smoking and age of 40-59 years $(\beta=1.10$, S.E.M. $=0.47, P=0.019)$ and age of $\geq 60$ years $(\beta=1.71$ s.E.M. $=0.70, P=0.014)$ respectively.

For taking the lifetime amount of tobacco consumption into account, we repeated all analyses for pack years as exposition variable. Among the 2448 individuals, there were 308 former or current smokers (160 men and 148 women), for which pack years could not be calculated due to missing values. Thus, 2140 subjects (1164 men and 976 women) were available for analysis on the association between pack years and thyroid volume progression, and 1423 subjects (740 men and 683 women) for the association between pack years and goiter respectively. Linear regression revealed a significant inverse association between pack years and thyroid volume progression in subjects aged 20-39 years $(\beta$ : -1.82 ; 95\% CI: $-2.16,-0.96, P<0.001)$. Other significant associations between lifetime amount of smoking and thyroid enlargement were not detected (data not shown).

\section{Discussion}

In the present study, we investigated the association of smoking with incident goiter and thyroid volume progression in a region of improved iodine supply. We detected age-dependent effects of smoking on the dependent variables. While there was an inverse association between smoking and goiter in younger subjects, a positive association was found between smoking and thyroid volume progression in older subjects.

West Pomerania is a region with former iodine deficiency. In the middle of the 1990s, the iodine supply was increased by means of iodized salt in food productions and individual salt consumption. This led to an increase in urinary iodine concentration (21) and to a decrease in goiter prevalence in adolescents living in the study region (22). Because sufficient iodine supply was only available for the past decade in West Pomerania, we observed the paradoxical situation of a high prevalence of goiter and other iodine deficiencyrelated disorders in a region of improved iodine supply (14). The results of the iodine monitoring in the KiGGS study (17) among children and adolescents demonstrated that Germany meanwhile has an improved

Table 3 Risk factors for incident goiter.

\begin{tabular}{|c|c|c|c|}
\hline & $\begin{array}{l}\text { 20-39 years odds ratio } \\
\text { (95\% confidence interval) }\end{array}$ & $\begin{array}{l}\text { 40-59 years odds ratio } \\
\text { (95\% confidence interval) }\end{array}$ & $\begin{array}{l}\text { 60-79 years odds ratio } \\
\text { (95\% confidence interval) }\end{array}$ \\
\hline Smoker at baseline and follow-up & $0.33(0.15 ; 0.71)^{*}$ & $1.17(0.62 ; 2.21)$ & $2.29(0.67 ; 7.77)$ \\
\hline Smoker at baseline only & $1.03(0.41 ; 2.57)$ & $2.19(0.89 ; 5.39)$ & $0.49(0.10 ; 2.33)$ \\
\hline Smoker at follow-up only & $0.57(0.19 ; 1.74)$ & $0.96(0.31 ; 3.00)$ & $0.68(0.07 ; 6.30)$ \\
\hline Smoker before baseline & $0.88(0.47 ; 1.64)$ & $1.12(0.69 ; 1.83)$ & $1.41(0.77 ; 2.58)$ \\
\hline Age (years) & $1.02(0.97 ; 1.07)$ & $1.00(0.97 ; 1.04)$ & $1.01(0.97 ; 1.06)$ \\
\hline Body mass index $\left(\mathrm{kg} / \mathrm{m}^{2}\right)$ & $1.12(1.06 ; 1.19)^{*}$ & $1.06(1.02 ; 1.11)^{*}$ & $1.10(1.04 ; 1.16)^{*}$ \\
\hline
\end{tabular}

Logistic regression for the incidence of goiter as outcome. Reference group for smokers: non-smokers. ${ }^{\star} P<0.05$. 
iodine supply at a lower recommended level. The present analysis revealed similar results. Due to the large study population, the slight decrease in the iodinecreatinine ratio over time attained statistical significance. Iodine excretion at follow-up, however, was still on a lower recommended level.

Smoking was inversely related with goiter in subjects $<40$ years and positively associated with thyroid volume progression in subjects $\geq 60$ years. Previous studies on the prevalence of goiter from formerly or currently iodine-deficient areas $(1,2,5,23)$ reported smoking as a risk factor for goiter whereas others did not $(7,24,25)$. The fact that the latter studies $(7,24$, 25 ) were only based on bivariate comparisons between smoking and goiter might have resulted in false conclusions because confounders (e.g. age) were not considered. Furthermore, our analysis that used lifetime amount of tobacco smoking as exposition variable confirmed the inverse association between increased amount of pack years and thyroid volume progression in the population $<40$ years. An association with goiter was not present in this subgroup. In the age group $\geq 60$ years, no interaction between pack years and thyroid enlargement was detected, which argues for an absence of an association between lifetime amount of tobacco smoking and goiter at older ages.

In line with these results, other studies $(10,11)$ conducted in an iodine-replete region did not detect any association between smoking and thyroid enlargement. This endorses the hypothesis that the association between smoking and thyroid enlargement is present in regions of iodine deficiency rather than in areas with sufficient iodine supply (4). In our analysis, smoking was a risk factor for thyroid volume progression only in the population $\geq 60$ years and, within this, only for those with goiter at baseline. After exclusion of these subjects, the association between smoking and thyroid change was no longer present. This finding suggests that individuals $\geq 60$ years are only affected by thyroid volume progression, if they already have developed goiter during time of iodine deficiency.

The goitrogenous effect of cigarette smoking can be partly explained by elevated plasma cyanate $\left(\mathrm{CN}^{-}\right)$ concentrations in smokers (26). Univalent anions with sizes similar to iodide, such as $\mathrm{CN}^{-}$, are able to competitively inhibit the transport of iodide into the thyroid gland. Our findings support the notion, that in regions with iodine deficiency this cohesion might fortify the development of goiter in smokers, whereas in regions with sufficient iodine supply smokers might be not that greatly affected (27).

In subjects $<40$ years, age was inversely associated with thyroid volume progression, but was not related to the risk of goiter. This finding supports the notion that, in general, younger individuals are less strongly affected by iodine deficiency than older individuals (14) and that this subpopulation particularly benefits from iodine fortification programs.
The definition of known thyroid disorders by selfreport, which was used as exclusion criterion, was certainly one limitation of the present study. Due to the vague symptoms of most thyroid diseases, the participant might be self-reported healthy, but suffer from biochemical thyroid disease. Therefore, we cannot fully rule out a certain misclassification in the chosen exclusion criterion.

We conclude that the inverse association between smoking and goiter in young adults and the lacking association of smoking with goiter and thyroid volume progression in adult non-goitrous subjects indicate that smoking has a declining impact on thyroid growth in the study region. Our findings mirror the improved iodine supply of Northeast Germany.

\section{Declaration of interest}

The authors declare that there is no conflict of interest that could be perceived as prejudicing the impartiality of the research reported.

\section{Funding}

The work is part of the Community Medicine Research net (CMR) of the University of Greifswald, Germany, which is funded by the Federal Ministry of Education and Research (grant no. ZZ9603), the Ministry of Cultural Affairs as well as the Social Ministry of the Federal State of Mecklenburg-West Pomerania. The CMR encompasses several research projects that are sharing data of the population-based Study of Health in Pomerania (SHIP; http://www.medizin.unigreifswald.de/icm/). Analyses were further supported by a grant of the German Research Foundation (DFG Vo 955/5-1). This research work was funded in part by grants from the German Federal Ministry for Education and Research, from the Ministry for Education, Research and Cultural Affairs and the Ministry for Social Affairs of the State Mecklenburg-West Pomerania.

\section{References}

1 Bartalena L, Bogazzi F, Tanda ML, Manetti L, Dell'Unto E \& Martino E. Cigarette smoking and the thyroid. European Journal of Endocrinology 1995133 507-512.

2 Bertelsen JB \& Hegedus L. Cigarette smoking and the thyroid. Thyroid 19944 327-331.

3 Galanti MR, Granath F, Cnattingius S, Ekbom-Schnell A \& Ekbom A. Cigarette smoking and the risk of goitre and thyroid nodules amongst parous women. Journal of Internal Medicine 2005 258 257-264.

4 Knudsen N, Laurberg P, Perrild H, Bulow I, Ovesen L \& Jorgensen T. Risk factors for goiter and thyroid nodules. Thyroid 200212 879-888.

5 Knudsen N, Bulow I, Laurberg P, Ovesen L, Perrild H \& Jorgensen T. Association of tobacco smoking with goiter in a low-iodine-intake area. Archives of Internal Medicine 2002162 439-443.

6 Völzke H, Schwahn C, Kohlmann T, Kramer A, Robinson DM, John U \& Meng W. Risk factors for goiter in a previously iodinedeficient region. Experimental and Clinical Endocrinology and Diabetes $2005113507-515$.

7 Olbricht T \& Hoff HG. Factors affecting thyroid gland volume. A contribution to the epidemiology of struma. Medizinische Klinik $198883279-284$.

8 Ericsson UB \& Lindgarde F. Effects of cigarette smoking on thyroid function and the prevalence of goitre, thyrotoxicosis and autoimmune thyroiditis. Journal of Internal Medicine 1991229 67-71. 
9 Christensen SB, Ericsson UB, Janzon L, Tibblin S \& Melander A. Influence of cigarette smoking on goiter formation, thyroglobulin, and thyroid hormone levels in women. Journal of Clinical Endocrinology and Metabolism 198458 615-618.

10 Barrere X, Valeix P, Preziosi P, Bensimon M, Pelletier B, Galan P \& Hercberg S. Determinants of thyroid volume in healthy French adults participating in the SU.VI.MAX cohort. Clinical Endocrinology 200052 273-278.

11 Gomez JM, Maravall FJ, Gomez N, Guma A \& Soler J. Determinants of thyroid volume as measured by ultrasonography in healthy adults randomly selected. Clinical Endocrinology $2000 \mathbf{5 3}$ 629-634.

12 Hansen PS, Brix TH, Bennedbaek FN, Bonnema SJ, Kyvik KO \& Hegedus L. Genetic and environmental causes of individual differences in thyroid size: a study of healthy Danish twins. Journal of Clinical Endocrinology and Metabolism $2004 \mathbf{8 9}$ 2071-2077.

13 Vestergaard P. Smoking and thyroid disorders - a meta-analysis. European Journal of Endocrinology 2002146 153-161.

14 Völzke H, Lüdemann J, Robinson DM, Spieker KW, Schwahn C, Kramer A, John U \& Meng W. The prevalence of undiagnosed thyroid disorders in a previously iodine-deficient area. Thyroid $200313803-810$.

15 Laurberg P, Jorgensen T, Perrild H, Ovesen L, Knudsen N, Pedersen IB, Rasmussen LB, Carle A \& Vejbjerg P. The Danish investigation on iodine intake and thyroid disease, DanThyr: status and perspectives. European Journal of Endocrinology $2006 \mathbf{1 5 5}$ 219-228.

16 John U, Greiner B, Hensel E, Lüdemann J, Piek M, Sauer S, Adam C, Born G, Alte D, Greiser E, Haertel U, Hense HW, Haerting J, Willich S \& Kessler C. Study of Health In Pomerania (SHIP): a health examination survey in an east German region: objectives and design. Sozial-und Präventivmedizin 2001 46 186-194.

17 Thamm M, Ellert U, Thierfelder W, Liesenkotter KP \& Volzke H. Iodine intake in Germany. Results of iodine monitoring in the German Health Interview and Examination Survey for Children and Adolescents (KiGGS). Bundesgesundheitsblatt, Gesundheitsforschung, Gesundheitsschutz $2007 \mathbf{5 0}$ 744-749.
18 Lorenz-Wawschinek O, Tiran B, Eber O \& Langsteger W. Photometric determination of iodine in urine. Experimental and Clinical Endocrinology 1994102 57-58.

19 Brunn J, Block U, Ruf G, Bos I, Kunze WP \& Scriba PC. Volumetric analysis of thyroid lobes by real-time ultrasound. Deutsche Medizinische Wochenschrift $1981 \mathbf{1 0 6} 409-414$.

20 Gutekunst R, Becker W, Hehrmann H, Olbricht W \& Pfannenstiel P. Ultrasonic diagnosis of the thyroid gland. Deutsche Medizinische Wochenschrift 1988113 1109-1112.

21 Meng W \& Scriba P. Jodversorgung in Deutschland, Probleme und erforderliche Maßnahmen: update (Iodine supply in Germany, problems and required measures: update 2002). Deutsches Ärzteblatt 200299 A2560-A2564.

22 Hampel R, Gordalla A, Zollner H, Klinke D \& Demuth M. Continuous rise of urinary iodine excretion and drop in thyroid gland size among adolescents in Mecklenburg-West-Pomerania from to 1997. Experimental and Clinical Endocrinology and Diabetes 1993108 197-201.

23 Hegedus L, Karstrup S, Veiergang D, Jacobsen B, Skovsted L \& Feldt-Rasmussen U. High frequency of goitre in cigarette smokers. Clinical Endocrinology 198522 287-292.

24 Berghout A, Wiersinga WM, Smits NJ \& Touber JL. Determinants of thyroid volume as measured by ultrasonography in healthy adults in a non-iodine deficient area. Clinical Endocrinology 1987 26 273-280.

25 Georgiadis E, Papapostolou C, Korakis T, Evagelopoulou K, Mantzoros C \& Batrinos M. The influence of smoking habits on thyroid gland volume: an ultrasonic approach. Journal of the Royal Society of Health 1997117 355-358.

26 Spitzweg C \& Morris JC. Sodium iodide symporter (NIS) and thyroid. Hormones $2002122-34$.

27 Brauer VF, Below H, Kramer A, Fuhrer D \& Paschke R. The role of thiocyanate in the etiology of goiter in an industrial metropolitan area. European Journal of Endocrinology $2006154229-235$.

Received 23 July 2008

Accepted 9 August 2008 